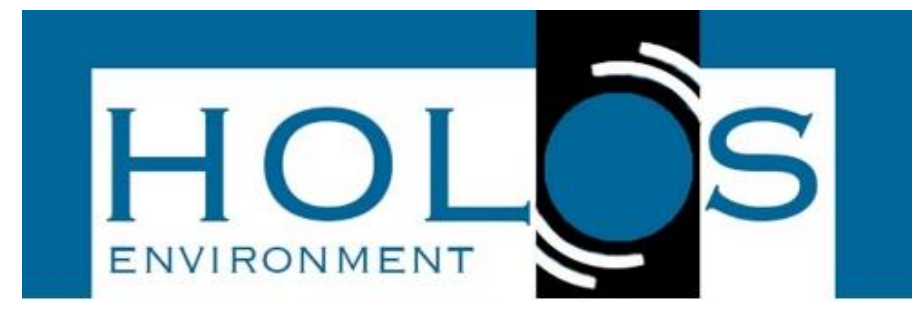

\title{
VIABILIDADE DE IMPLANTAÇÃO DE UM BIODIGESTOR EM UMA GRANJA DE SUÍNOS
}

\section{VIABILITY OF IMPLANTATION OF A BIODIGESTOR IN A SWINE FARM}

\author{
Fernando Pasini ${ }^{1}$; Aline Ferrão Custódio Passini ${ }^{1}$; Andressa Aparecida Piovezan ${ }^{1}$; \\ Daiana Correa e Silva ${ }^{1}$; Giovanna Angelina Machado ${ }^{1}$
}

Artigo recebido em: 30/03/2018 e aceito para publicação em: 12/04/2019.

DOI: http://dx.doi.org/10.14295/holos.v19i1.12278

Resumo: A produção suinícola é uma das atividades agropecuárias mais importantes da Região Sul brasileira, no entanto é responsável por gerar grande quantidade de dejetos e resíduos. Dentre as formas mais utilizadas para o tratamento destes dejetos estão os sistemas de lagoas e a biodigestão, sendo o segundo o mais rentável, pelo potencial de produção energética e qualidade do biofertilizante gerado. Considerando que o maior empecilho para implantação de sistemas de biodigestor seja os custos de instalação, este estudo objetivou caracterizar uma unidade produtora de leitões (UPL), dimensionar os parâmetros básicos de um biodigestor e extrapolar o tempo necessário para o retorno dos custos de investimento. A unidade escolhida localiza-se no Município de Vista Alegre/RS, mantém uma criação média de 9.964 suínos, o que resulta diariamente em um total de $47,76 \mathrm{~m}^{3}$ de dejetos. Com base neste volume será necessário a implantação de um biodigestor com no mínimo $1.671,6 \mathrm{~m}^{3}$ de capacidade. A estimativa de investimento inicial para a implementação do projeto é de $\mathrm{R} \$ 251.761,40$ reais. E a produção diária de biogás é de $11.411,7 \mathrm{~m}^{3}$, com capacidade para gerar até $18.405,96 \mathrm{KWh}$, assim estimou-se um tempo de retorno do investimento de três anos. Considerandose portanto, viável a instalação.

Palavras-chave: Suinocultura. Biodigestão. Bioenergia.

Abstract: Pig production is one of the most important agricultural activities in the Brazilian South Region, however it is responsible for generating large amounts of waste and wastes. Among the most used forms for the treatment of these wastes are lagoon systems and biodigestion, the second one being the most profitable, due to the energy production potential and the quality of the biofertilizer generated. Considering that the greatest impediment to the implantation of biodigester systems is the installation costs, this study aimed to characterize a pig production unit (UPL), to size the basic parameters of a biodigester and to extrapolate the time required for the return of the investment costs. The chosen unit is located in the Municipality of Vista Alegre / RS, maintains an average of 9,964 pigs, which results in a total of $47.76 \mathrm{~m}^{3}$ of waste. Based on this volume will be necessary the implantation of a biodigestor with at least $1,671.6 \mathrm{~m}^{3}$ of capacity. The initial investment estimate for the implementation of the project is $R \$ 251,761.40$. And the daily production of biogas is $11,411.7 \mathrm{~m}^{3}$, with a capacity to generate up to $18,405.96 \mathrm{KWh}$, thus it was estimated a time of return of investment of three years. Considering therefore, the installation feasible.

Palavras-chave: Swine breeding; Biodigestion; Bioenergy.

\footnotetext{
${ }^{1}$ Universidade Federal de Santa Maria (UFSM). Santa Maria, RS. E-mails: (fernando pasini@hotmail.com, alinefcustodio@gmail.com, andressapiovezan@hotmail.com, daianacorrea.cs@gmail.com, giovannaangelinam@gmail.com)
} 


\section{INTRODUÇÃO}

A criação de suínos possui um importante papel dentre as atividades pecuárias do Brasil, gera emprego, recursos financeiros e fixa o homem no campo, além de fornecer alimentos de alto valor nutritivo para o consumo da população. O Brasil classifica-se mundialmente como o terceiro maior produtor de carne suína, correspondendo a $4 \%$ da produção mundial, oriundas de um rebanho nacional quantificado em 40.332 .553 cabeças (GERVÁSIO, 2017).

No Brasil a suinocultura caracteriza-se majoritariamente, em criação do tipo semi-intensivo e intensivo, e em menor escala criações do tipo extensiva. É uma atividade desenvolvida predominantemente em pequenas propriedades rurais, sendo mais de $80 \%$ da produção oriunda de unidades com até 100 hectares (SCHIMIDT, GOTTARDI e NADVORNY, 2007; MACEDO, 2013).

A região sul excepcionalmente destaca-se na produção de suínos em confinamento, possuindo uma forte cadeia produtiva, que vai desde a produção de ração ao abate dos animais (SCHIMIDT, GOTTARDI e NADVORNY, 2007), sendo os estados desta região os maiores produtores nacionais, em primeiro lugar o Estado do Paraná ( 7.134 .055 cabeças), em segundo Santa Catarina (6.775.869 cabeças) e em terceiro o Rio Grande do Sul (5.928.885 cabeças) (GERVÁSIO, 2017).

A concentração da produção apesar de favorável à economia regional, aumentando a distribuição de renda e consequente poder de compra, torna-se um desafio à proteção ambiental, principalmente em relação ao manejo e tratamento dos dejetos gerados (SERAFIN e GUIMARÃES FILHO, 2011; PINTO et al., 2014).

Os sistemas agropecuários dão origem a vários tipos de resíduos orgânicos que, quando corretamente manejados e utilizados, convertem-se em ótimas fontes de nutrientes para os solos agrícolas (KONZEN e ALVARENGA, 2007).

O dejeto suíno é formado por restos de ração, fezes, urina e água de lavagem das baias, é rico em nitrogênio, fósforo, potássio, cálcio, magnésio, enxofre e cobre (CARDOSO; OYAMADA e SILVA, 2015). Os nutrientes contidos nos dejetos têm alto valor agregado, sobretudo quando considerado o aumento que o preço dos fertilizantes químicos tem sofrido nos últimos anos (KUNZ, HIGARASHI e OLIVEIRA, 2005).

O tratamento do dejeto suíno visa promover a decomposição da matéria orgânica, a qual ocorre por meio de processos biológicos aeróbios, anaeróbios ou fa- 
cultativos (FERNANDES e OLIVEIRA, 1995). Dentre as formas mais comuns de tratamento estão a compostagem, a sobreposição de cama, a separação (sólidoliquido), utilização de sistemas de lagoas e a biodigestão (MAPA, 2016).

Os sistemas de biodigestão são muito interessantes do ponto de vista econômico e ambiental, visto que, quando utilizados, além da produção do biofertilizante (como nas demais técnicas) é possível o aproveitamento energético dos gases gerados no processo de biodigestão anaeróbia (BARICHELLO et al., 2015). Dentre os principais gases produzidos pela digestão do dejeto estão o metano $\left(\mathrm{CH}_{4}\right)(54-80$ \%), o dióxido de carbono $\left(\mathrm{CO}_{2}\right)(20$ a $45 \%)$ o sulfeto de hidrogênio $\left(\mathrm{H}_{2} \mathrm{~S}\right)(3 \%)$ e o gás nitrogênio $\left(\mathrm{N}_{2}\right)(3 \%)$ (MAPA, 2016).

Ainda que o MAPA (2016) recomende que este tipo de sistema pode ser utilizado em granjas de qualquer tamanho, uma questão muito debatida refere-se à viabilidade de instalação, custos de operação, manutenção e tempo de retorno de investimento.

Desta forma esta pesquisa teve por objetivo caracterizar uma unidade produtora de leitões (UPL), dimensionar os parâmetros básicos de um biodigestor e extrapolar o tempo necessário para o retorno dos custos investidos em um sistema de biodigestão anaeróbia.

\section{MATERIAIS E MÉTODOS}

A metodologia foi dividida em duas partes, a primeira consistiu na caraterização da propriedade: Para isso foram realizadas visitas técnicas e entrevista com o proprietário para que fosse possível conhecer a propriedade e face a isto estipular as necessidades energéticas diárias e a quantidade de animais existentes, obtendose uma média diária de dejetos gerados.

Para a segunda etapa foram consultadas referências na literatura para o cálculo das dimensões necessárias ao biodigestor, assim como os cálculos do potencial de geração de biogás e custos de implantação.

\subsection{Caracterização da propriedade}

O local escolhido para a pesquisa é uma granja UPL localizada na linha Piaia/ Centenaro / Pessoto, s/n - Km 03 - Zona Rural, no Município de Vista Alegre-RS 
(27²2'9.33"S, 53²8'20.11"O).

A granja possui 2100 matrizes alojadas em: Um prédio para matrizes de reposição (1.553,12 m²); Dois prédios para Matrizes de Gestação (3.106,24 m²); Dois prédios para Matrizes em Maternidade (2.698,24 m²); e Dois prédios para 6500 Leitões em Creche $\left(2.698,24 \mathrm{~m}^{2}\right)$.

Além de um escritório com 207,69 $\mathrm{m}^{2}$ e uma unidade de compostagem com $107,87 \mathrm{~m}^{2}$, totalizando $10.618,92 \mathrm{~m}^{2}$ de área construída. Tudo isso em uma área rural de 14,4 ha (FIGURA 1).

Figura 1- Mapa de localização da granja

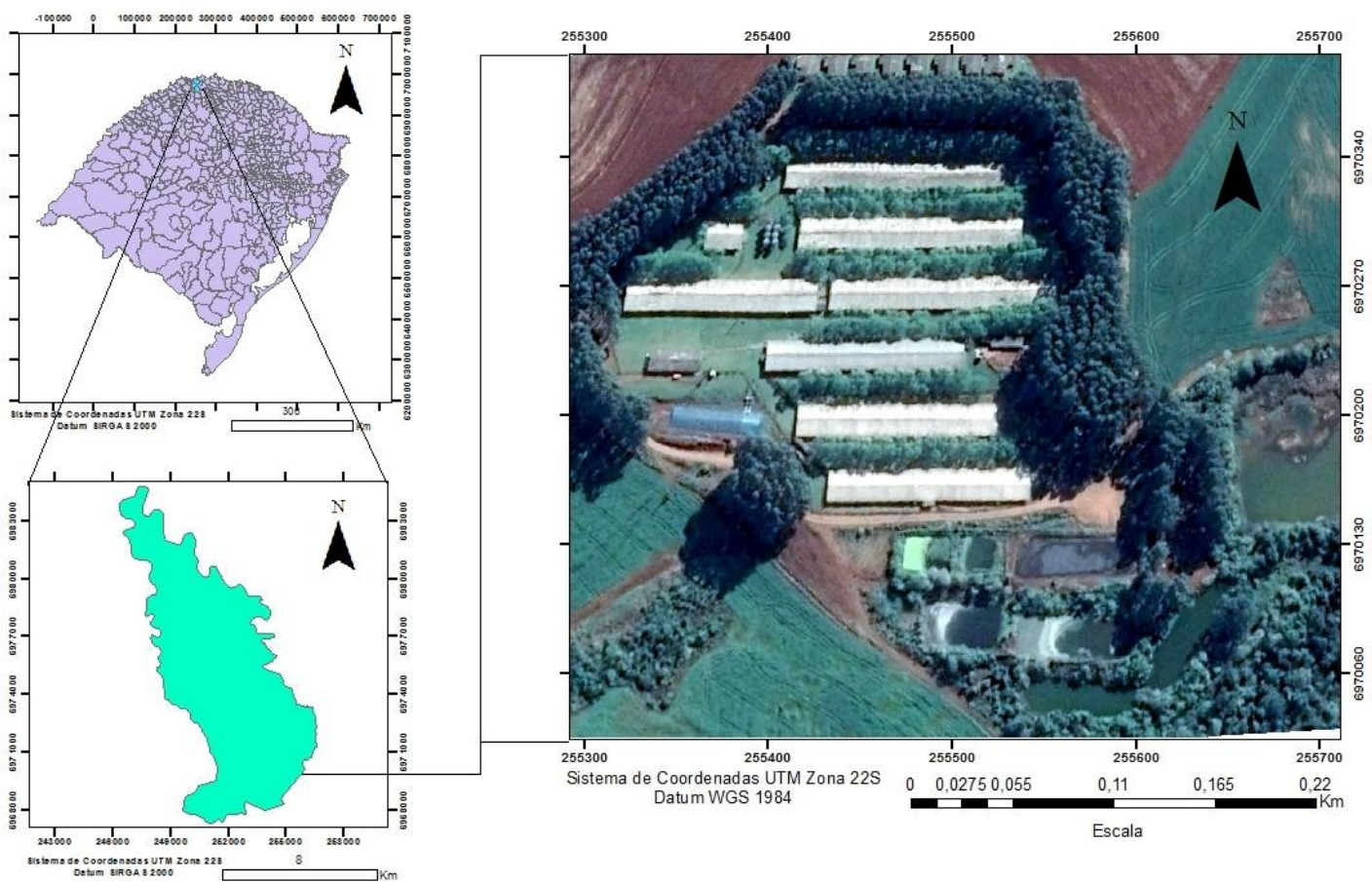

Fonte: Construído a partir de IBGE (2010)

\subsection{Capacidade da produção de biogás}

Para cálculos da capacidade de produção de biogás na propriedade foi utilizado fórmulas mencionadas por Farret (2010) assim temos:

$$
T_{\text {gás/animal }}=m_{\text {dejeto/dia }} x f_{\text {Produçâo/animal }}
$$

Onde:

$T_{\text {gás/animal }}=$ Total de Gás produzindo por um animal por dia $\left(\mathrm{m}^{3} \cdot\right.$ animal ${ }^{-1} \cdot$ dia $)$ 
$m_{\text {dejeto/dia }}=$ Massa de dejetos produzido por um animal por dia $\left(\mathrm{kg} \cdot\right.$ animal $\left.^{-1} \cdot \mathrm{dia}^{-1}\right)$

$f_{\text {produção/animal }}=$ Quantidade de biogás gerado por $1 \mathrm{~kg}$ de dejeto. $\left(\mathrm{m}^{3} \cdot \mathrm{kg}^{-1}\right)$

$$
T 1_{\text {gás } / \text { dia }}=T_{\text {gás } / \text { animal }} x N
$$

Onde:

$T 1_{\text {gás/dia }}=$ Quantidade de gás diário gerado na propriedade $\left(\mathrm{m}^{3}\right.$.animal ${ }^{-1}$.dia $)$

$N=$ Número de animais da propriedade (animal)

Para calcular a capacidade de produção de biogás das propriedades, foi utilizada média ponderada:

$C P_{\text {Biogás }}=\frac{(K g 1 \times K g 2)+(K g 3 \times K g 2)+(K g 4 \times K g 2)}{T 1_{g a ́ s} / d i a}$

Onde:

$K g 1=$ Média de dejetos produzidos por suínos de engorda $(\mathrm{kg})$

$K g 2=$ Média de dejetos produzidos por suínos UPL $(\mathrm{kg})$

$K g 3=$ Média de dejetos produzidos por suínos de maternidade $(\mathrm{kg})$

Kg4= Média de dejetos produzidos por suínos de matriz $(\mathrm{kg})$

Para quantificar o volume de dejeto gerado por animal em cada fase (Tabela 1), foram utilizados os valores encontrados por Oliveira (1993).

Tabela 1- Volume de dejeto produzido por suíno em cada fase de desenvolvimento

\begin{tabular}{lccc}
\hline Categoria & Esterco $\mathbf{~ ( k g . d i a ~}^{-1}$ ) & $\begin{array}{c}\text { Esterco + urina } \\
\left.\text { (kg.dia }^{-1}\right)\end{array}$ & $\begin{array}{c}\text { Dejetos Líquidos } \\
\left.\text { (.dia }^{-1}\right)\end{array}$ \\
\hline Suínos 25 a 100 kg & 2,30 & 4,90 & 7,00 \\
Porcas em Gestação & 3,60 & 11,00 & 16,00 \\
Porcas Lactação + Leitões & 6,40 & 18,00 & 27,00 \\
Cachaço & 3,00 & 6,00 & 9,00 \\
Leitões na creche & 0,35 & 0,95 & 1,40 \\
Média & 2,35 & 5,80 & 8,60 \\
\hline
\end{tabular}

Fonte: Oliveira (1993)

Após realizados os cálculos foi elaborado um quadro para analisar todos os gastos de energia da propriedade, e assim ter ciência de como distribuir a energia a ser gerada. 


\subsubsection{Dimensionamento do biodigestor}

Os cálculos de dimensionamento do biodigestor, foram baseados na metodologia utilizada por Junqueira (2014). Assim:

$$
V B=V C x T D H
$$

Onde:

$V B=$ Volume do biodigestor (dejetos + água $)-\left(\mathrm{m}^{3} \cdot \mathrm{dia}^{-1}\right)$;

$V C=$ Volume carga diária $\left(\mathrm{m}^{3} \cdot \mathrm{dia}^{-1}\right)$;

$T D H=$ Tempo de Detenção Hidráulica (dia)

O tempo de detenção hidráulica utilizado para dejetos de suínos é de em média 35 dias, sendo este o tempo utilizado para o cálculo (OLIVEIRA, 2004).

Para o cálculo do volume de biogás produzido, foi utilizada a relação de dejeto/biogás de que a cada $1 \mathrm{~kg}$ de dejeto suíno injetado no biodigestor são gerados $0,35 \mathrm{~m}^{3}$ de biogás (TAKAMATSU e OLIVEIRA, 2002)

O cálculo de energia elétrica gerada, considera que para produzir $1 \mathrm{kWh}$ são necessários $0,62 \mathrm{~m}^{3}$ de biogás, sendo a eficiência de conversão do biogás em energia elétrica em grupos geradores de aproximadamente $25 \%$ (AISSE e OBLADEN, 1982).

\section{RESULTADOS E DISCUSSÃO}

A granja escolhida para estudo é classificada como de grande porte (possuindo mais de 100 matrizes) (BONETT e MONTICELLI, 1998). Possui uma ótima estrutura, abriga 9.964 suínos, que produzem um total de $47,76 \mathrm{~m}^{3}$ de dejetos diariamente (Tabela 1). O sistema de tratamento utilizado para os dejetos são as lagoas de estabilização e o reaproveitamento de resíduo é feito por incorporação no solo, como biofertilizante. 
Tabela 2- Levantamento do número de animais e estimativa de produção diária de dejetos

\begin{tabular}{lcccc}
\hline Fase & № animais & $\begin{array}{c}\text { Dejetos líquidos por } \\
\text { animal (I.dia }\end{array}$ & $\begin{array}{c}\text { Total }) \\
\left(\text { I.dia }^{-1}\right)\end{array}$ & $\begin{array}{c}\text { Total } \\
\left(\mathbf{m}^{3} . \text { dia }^{-1}\right)\end{array}$ \\
\hline Leitoas (25 a 100 kg) & 170 & 7 & 1190 & 1,19 \\
Gestantes & 1786 & 16 & 28576 & 28,58 \\
Lactantes & 265 & 27 & 7155 & 7,16 \\
Creche & 7743 & 1,4 & 10840 & 10,84 \\
Total & 9964 & 51,4 & 47761 & 47,76 \\
\hline
\end{tabular}

Atualmente as principais matrizes energéticas utilizadas para suprir as necessidades da granja são a eletricidade e a madeira (Tabela 3). A energia elétrica é proveniente da concessionária local, sendo necessário em média anualmente 248.275,86 KWh, já a madeira utilizada é adquirida de extração madeireira de terceiros, sendo necessário em média 1000 t anuais.

Estas fontes juntas somam um montante de $\mathrm{R} \$ 177.000,00$ de despesa energética, que é utilizada principalmente para iluminação de toda a estrutura, aquecimento das baias da creche em períodos de baixas temperaturas e funcionamento do sistema de alimentação automático.

Tabela 3- Custo e consumo total anual com fontes energéticas

\begin{tabular}{ccc}
\hline Descriminação & Consumo anual & Custo Anual (R\$) \\
\hline Energia elétrica & $248.275,86 \mathrm{kWh}$ & $144.000,00$ \\
Madeira & $1000 \mathrm{t}$ & $33.000,00$ \\
Total & & $177.000,00$ \\
\hline
\end{tabular}

Fonte: Documentos fornecidos pelo proprietário

A partir do total de dejetos produzidos diariamente, estimou-se através da metodologia adotada por Junqueira (2014), que será necessária a implantação de um biodigestor com no mínimo 1671,6 $\mathrm{m}^{3}$ de capacidade. Considera-se, através da metodologia adotada por Junges et al. (2009), que o investimento inicial para a implementação do projeto seja de $R \$ 251.761,40$.

Apesar do grande volume demandado pelo biodigestor, este terá dimensões subjetivas ao modelo escolhido, podendo ser enterrado ou ao nível do solo (FUKUDA, 2013). De qualquer forma, a propriedade dispõe de 13,4 há não construídas, utilizadas para produção de grãos, sendo que parte desta pode ser cedida a instalação do biodigestor.

A quantidade prevista de biogás produzido diariamente, será de $11.411,7 \mathrm{~m}^{3}$, com capacidade para gerar 18.405,96 KW. Assim, anualmente, poderão ser produzidos cerca de 4.165.270,5 $\mathrm{m}^{3}$ de biogás, e capacidade de geração média de 
6.718.175,4 KWh, o que será suficiente para tornar a granja autossuficiente em energia. A produção de biogás excederá o necessário para a granja assim torna-se necessário a utilização de um sistema de exaustão do gás e sua posterior queima, para isso é preciso realizar a instalação de um flare, que serve como uma chaminé, o qual mantém-se aceso em virtude da queima da gás extra que não é utilizado pelo gerador.

Considera-se nesta simulação, que os investimentos relativos ao sistema de biodigestores sejam realizados pela própria granja. A receita da simulação (Tabela 3) foi estimada somente através da economia gerada na propriedade no quesito energético.

Tabela 4- Fluxo líquido de caixa do projeto para um horizonte de planejamento de cinco anos

\begin{tabular}{|c|c|c|c|c|}
\hline \multicolumn{5}{|c|}{ Fluxo de caixa financeiro do projeto - sem financiamento } \\
\hline Ano & Investimento & $\begin{array}{l}\text { Margem Ope- } \\
\text { racional }\end{array}$ & $\begin{array}{c}\text { Fluxo Líquido } \\
\text { de Caixa }\end{array}$ & $\begin{array}{l}\text { Fluxo Líquido de } \\
\text { Caixa Acumulado }\end{array}$ \\
\hline 1 & 251761,4 & - & $-251761,40$ & $-251761,40$ \\
\hline 2 & & 177000,00 & 177000,00 & $-74761,40$ \\
\hline 3 & & 177000,00 & 177000,00 & 102238,00 \\
\hline 4 & & 177000,00 & 177000,00 & 279238,00 \\
\hline 5 & & 177000,00 & 177000,00 & 546238,6 \\
\hline Total & & & & 601191,8 \\
\hline
\end{tabular}

Com base no fluxo líquido de caixa do projeto, tem-se que o tempo de retorno dos investimentos realizados gira em torno de três anos. Para um horizonte de planejamento de cinco anos, a receita total obtida é de $\mathrm{R} \$ 601.191,00$ proporcionando uma economia anual de $\mathrm{R} \$ 177.000,00$.

\section{CONCLUSÕES}

O trabalho demonstrou ser viável a implantação de um biodigestor na granja de estudo. Porém a grande produção diária de dejetos, irá demandar de uma grande área para a construção.

Sabendo-se que a granja usa anualmente cerca de 248.275,86 KWh, concluise que a geração de energia a partir da produção de biogás é capaz de tornar a propriedade autossuficiente em energia, suprindo todas as necessidades internas. 
Além da produção energética a decomposição de material orgânico em biodigestores gera compostos que podem ser utilizados para adubação agrícola e assim diminuindo ainda mais os custos de operação.

\section{REFERÊNCIAS}

AISSE, M. M.; OBLADEN, N. L. Tratamento de esgotos por biodigestão anaeróbia. Curitiba: CNPq. ITAH/IPPUC, PUCPR, p.99. 1982.

BARICHELLO, R.; HOFFMANN, R.; SILVA, S. O. C.; DEIMLING, M. F.; CASAROTTO FILHO, N. O uso de biodigestores em pequenas e médias propriedades rurais com ênfase na agregação de valor: um estudo de caso na Região Noroeste do Rio Grande do Sul. Revista em Agronegócio e Meio Ambiente, Maringá (PR). V. 8 n 2. 2015.

BONETT, L. P., MONTICELLI, C. J. Suínos: o produtor, a Embrapa responde. Brasília: Embrapa-SPI, Concórdia: Embrapa Suínos e Aves, 243 p, 2009.

BONETT, L. P.; MONTICELLI, C. J. O produtor pergunta, a Embrapa responde. 2. ed., rev.- Brasília, DF. Embrapa-SPI; Concórdia: Embrapa Suínos e Aves, 1998.

CARDOSO, B. F.; OYAMADA, G. C.; SILVA, C. M. Produção, tratamento e uso dos dejetos suínos no brasil. Desenvolvimento em Questão. Editora Unijuí, n. 32. out./dez. 2015.

FUKUDA, J. C. Projeto de instalação de um biodigestor de baixo custo na sede de uma unidade de conservação: saneamento, aproveitamento energético e demonstração de alternativa sanitária para a região. Monografia (Pós-Graduação em Formas Alternativas de Energia). Universidade Federal de Lavras - UFLA- Lavras MG, 2013, 51p.

FARRET, F. A. Aproveitamento de pequenas fontes de energia elétrica. 2. ed. Santa Maria - RS. Editora UFSM, 2010.

FERNANDES, C. O. M.; OLIVEIRA, P. A. V. Armazenagem de dejetos suínos. In: Aspectos práticos de dejetos de suínos. Florianópolis: EPAGRI, EMBRAPA-CNPSA, 1995. p. 45-68, 1995.

GERVÁSIO, E. W. Suinocultura Paranaense. Secretaria de Estado da Agricultura e do Abastecimento - SEAB, Departamento de Economia Rural. Curitiba -PR, 2017.

GOOGLE. Google Earth. Version Pro. 2015. Imagens da unidade produtora de leitões. Disponível em: https://www.google.com.br/earth/download/gep/agree.html. Acesso em: 10 nov. 2017.

IBGE. INSTITUTO BRASILEIRO DE GEOGRAFIA E ESTATÍSTICA. Downloads: cartas do ano de 2010. Rio de Janeiro: Instituto Brasileiro de Geografia e Estatística. Disponível em: https://downloads.ibge.gov.br/downloads_geociencias.htm. Acesso em: 30 mar. 2018.

JUNQUEIRA, S. L. C. D. Geração de energia através de biogás proveniente de esterco bovino: estudo de caso na fazenda aterrado. Monografia (Graduação em Engenharia Mecânica) Universidade Federal do Rio de Janeiro (UFRJ), Rio de Janeiro-RJ, 55p. 2014.

KONZEN, E. A.; ALVARENGA, R. C. Cultivo do milho: fertilidade de solos, adubação orgânica. Embrapa Milho e Sorgo, 3. ed. 2007 
KUNZ, A.; HIGARASHI, M. M.; OLIVEIRA, P. A. Tecnologias de manejo e tratamento de dejetos de suínos estudadas no Brasil. Cadernos de Ciência \& Tecnologia, Brasília, v. 22, n. 3, p. 651-665, 2005.

MACEDO, F. J. Dimensionamento de biodigestores para tratamento de dejetos da produção suína. Monografia (Graduação em Engenharia Sanitária e Ambiental) - Universidade Federal de Santa Catarina (UFSC). Florianópolis-SC, 117p, 2013.

MINISTÉRIO DA AGRICULTURA, PECUÁRIA E ABASTECIMENTO (MAPA). Suinocultura de baixa emissão de carbono: tecnologias de produção mais limpa e aproveitamento econômico dos resíduos da produção de suínos. Brasília. 2016.

OLIVEIRA, P. A. V. Manual de manejo e utilização dos dejetos de suínos. Concórdia: EMBRAPA/ CNPSA (Documento, 27), 188 p. 1993.

OLIVEIRA, P. A. V. Tecnologias para o manejo de resíduos na produção de suínos: manual de boas práticas. Embrapa Suínos e Aves. Concórdia, 2004.

PINTO, L. P.; CABRAL, A. C.; SCHNEIDER, L. T.; AZEVEDO, K. D.; FRIGO, J. P.; FRIGO, E. P. Levantamento de dados sobre os dejetos suínos e suas características. Revista Brasileira de Energias Renováveis. v. 3, p. 179-187, 2014.

SCHMIDT, V.; GOTTARDI, C. P. T.; NADVORNY, A. Segurança sanitária durante a produção, o manejo e a disposição final de dejetos de suínos. In: SEGANFREDO, M.A. (Ed.) Gestão ambiental na suinocultura. Brasília: Embrapa Informação Tecnológica, 302 p., cap. 11, p. 259-286, 2007.

SERAFIN, G. B.; GUIMARÃES FILHO, L. P. Estudo sobre o reaproveitamento dos dejetos de suínos na bacia do rio Sangão - Santa Catarina. In: ENCONTRO DE ECONOMIA CATARINENSE: CRESCIMENTO E SUSTENTABILIDADE, 5, Anais...2011. Florianópolis, 2011.

JUNGES, D. M; KLEINSCHMITT, S. C; SHIKIDA, P. F. A; SILVA, J. R. Análise econômicofinanceira da implantação do sistema de biodigestores no município de Toledo - Paraná.

Revista de Economia, v. 35, n. 1, 2009.

TAKAMATSU, A. A; OLIVEIRA, R. F. Manual de biossistemas integrados na suinocultura. Curitiba: TECPAR, 2002. 